\section{The Watching Eyes Effect on Charitable Donation is Boosted by Fewer People in the Vicinity}

\author{
Ryo Oda*, Ryota Ichihashi \\ Nagoya Institute of Technology, Nagoya 466-8555, Japan \\ *Author for correspondence (oda.ryo@nitech.ac.jp)
}

Previous field experiments have found that artificial surveillance cues facilitated prosocial behaviors such as charitable donations and littering. Several previous field studies found that the artificial surveillance cue effect was stronger when few individuals were in the vicinity; however, others reported that the effect was stronger in large groups of people. Here, we report the results of a field study examining the effect of an artificial surveillance cue (stylized eyes) on charitable giving. Three collection boxes were placed in different locations around an izakaya (a Japanese-style tavern) for 84 days. The amount donated was counted each experimental day, and the izakaya staff provided the number of patrons who visited each day. We found that the effect of the stylized eyes was more salient when fewer patrons were in the izakaya. Our findings suggest that the effect of the artificial surveillance cue is similar to that of "real" cues and that the effect on charitable giving may weaken when people habituate to being watched by "real" eyes.

\section{Keywords}

prosociality, watching eyes effect, charitable giving, donation, collection box

\begin{abstract}
Introduction
Several laboratory and field studies have shown that artificial surveillance cues increase prosocial behavior toward unrelated strangers, a phenomenon known as the "watching eyes effect" (e.g., Oda, Niwa, Honma, \& Hiraishi, 2011). However, other studies have found no effect of eye images on behavior (e.g., Matsugasaki, Tsukamoto, \& Ohtsubo, 2015). Northover, Pedersen, Cohen, and Andrews (in press) conducted two metaanalyses of the effect of artificial observation cues on generosity and concluded that artificial surveillance cues do not increase how generous an individual is or the probability that individuals will show generosity. Although their investigation was restricted to laboratory experiments using economic games, the results suggest that the watching eyes effect is not robust and is situation
\end{abstract}

dependent

The number of people in the vicinity may have an effect on the watching eyes effect. If artificial observation cues heighten the feeling of being watched, it may be that "real" eyes in the vicinity also evoke this feeling. Field studies are more appropriate than laboratory studies for investigation of the "real" eyes factor. Two previous studies using collection boxes found that the watching eyes effect was stronger when fewer people present. Powell, Roberts, and Nettle (2012) placed containers at six checkout counters in a supermarket in the United Kingdom to collect donations for a charity. They attached an image of eyes on half of the containers and an image of three stars on the remaining half. During relatively quiet weeks, the containers with eyes received $59 \%$ more amount of donated per thousand customers than did the control containers. In contrast, during relatively busy weeks, the containers with eyes received only $28 \%$ more donations than the control containers. Ekström (2012) used recycling machines located in 38 stores of a Swedish supermarket chain to test the effect of watching eyes over a 12-day period. Customers who used the machines to recycle cans and bottles were given the choice of keeping the money received or donating it to a charitable organization. An image of eyes was posted on the recycle machines for the first 6 days, and a control image of flowers was displayed on the remaining 6 days. Although there was no general effect of eyes, on days when few customers visited the store, the author found a $30 \%$ increase in the amount donated under the eye compared with the control condition. Furthermore, the findings of a field study investigating norm compliance behavior were consistent with those of Ekström (2012). Ernest-Jones, Nettle, and Bateson (2011) compared littering behavior in the presence of wall posters featuring eyes and posters featuring flowers in a university cafeteria in the United Kingdom. The authors found that litter was left on fewer cafeteria tables under the eyes compared with the flowers condition, and the effect was greater when fewer people were in the cafeteria. However, a subsequent field study found that the presence of eyes had the opposite effect on littering behavior. Bateson, Callow, Holmes, Redmond Roche, and Nettle (2013) found that large signs displaying images of watching eyes posted near university bicycle racks in the United Kingdom tended to prevent littering only when a large number of people (six or more) were in the immediate vicinity.

Bateson et al. (2013) argued that the discrepant results could be explained by methodological differences: their sign was larger than that used in other studies, and their experiment was conducted outside in a public space where participants were passing through as opposed to inside, as in a supermarket. Indeed, Bateson et al. (2015) conducted two experiments using smaller watching eyes stimuli displayed on litter itself, and found that the watching eyes effect was larger when no other individuals were in the vicinity. However, charitable donation is not the same as 
the desire to avoid violating norms. For example, Oda, Kato, and Hiraishi (2015) compared the tendency to tell prosocial lies in the presence and absence of stylized eyes. Participants tended to tell lies that benefitted others under the control condition, whereas the tendency toward prosocial lying disappeared under the watching eyes condition. These findings suggest that donating money and littering should be considered separately. With regard to donations, the presence of fewer people in the vicinity increased prosocial behavior. However, only two studies have investigated customer behavior in stores. Further studies are needed to clarify the effect of watching eyes under different conditions and in various group sizes.

We investigated the effect of the number of individuals in the vicinity on charitable donations in an izakaya (a Japanese-style tavern) setting. Oda and Ichihashi (2016) used transparent collection boxes to investigate the effect of eye images and local norms (controlled by money visible in a collection box) on charitable donations. They found that the amount donated was greater under the large- than under the small-norm condition. The presence of eye images increased the overall amount donated, but was more salient under the small-norm condition. Oda and Ichihashi (2016) treated the number of patrons who visited the izakaya each day as an offset item in their general linear model, and the effect on amount of donation was not directly considered because their study was a replication of Fathi, Bateson, and Nettle (2014) which investigated effects of norms, watching eyes and their interaction. In this study we used the same dataset by Oda and Ichihashi (2016) but employed a different model considering effects of the number of patrons, eyes images and their interaction on the amount of donation.

\section{Methods}

Three ready-made transparent collection boxes with a money slot (Plasart, Inc.; $120 \times 97 \times 179 \mathrm{~mm}, \mathrm{~W} \times \mathrm{D} \times$ $\mathrm{H}$; Figure 1) were placed in three locations around an izakaya located near a terminal station in Nagoya, Japan. A card on the front of the box indicated that the donation would be sent to the United Nations High Commissioner for Refugees (UNHCR) to aid refugees. On the back, we inserted a white card $(100 \times 170 \mathrm{~mm}, \mathrm{~W} \times \mathrm{H})$ with a brief written explanation of the refugee crisis, with the watching eyes stimulus image $(50 \times 30 \mathrm{~mm}, \mathrm{~W} \times \mathrm{H})$ in the upper portion. The control stimulus was a reconstructed version of the same image, but the eyes were not identifiable (Figure 1). Under the small-norm condition, we placed 150 coins in the box including $¥ 1, ¥ 10, ¥ 50$, and $¥ 100$, amounting to a total value of 4,050 JPY. Under the large-norm condition, the box contained 149 coins including $¥ 1$, $¥ 10, ¥ 50$, $¥ 100$, and $¥ 500$, and a $¥ 1,000$ banknote amounting to a total value of 15,930 JPY.

The boxes were left in place for 84 days between November 1, 2015, and February 19, 2016. One of the four combinations of the eyes/control and small-/large-norm conditions was randomly allocated to each day during the study period; thus, each combination was tested for a total of 21 days. The izakaya was open between 5:30 $\mathrm{pm}$ and 12:30 am. Each day during the experimental period, the collection boxes were emptied by an author or an izakaya staff member after the izakaya closed, and the amount donated was counted. The staff provided the number of patrons and groups who visited the izakaya on each experimental day (see Oda \& Ichihashi [2016] for a detailed description of the methods).

\section{Results}

A total of 10,174 JPY was donated, with a median amount of 90 JPY per day (range: $¥ 0-530$ ). In total, 8,269 patrons visited the izakaya during the experiment, and the median number of patrons was 92.5 (range: $46-176$ patrons). The largest daily donation, $530 \mathrm{JPY}$, was made under the largenorm/no-eyes condition. We considered this donation an outlier and excluded it from the analyses because the amount was 1.5 times the interquartile range (202.1 JPY) above the third quartile (178.5 JPY). A total of 8,189 patrons visited the izakaya during the remaining 83 experimental days, and the median number of patrons per day was 93 (range: 46-176). The total amount donated was 9,644 JPY (see Oda \& Ichihashi [2016] for details).

A Poisson regression model was fitted using the

b)

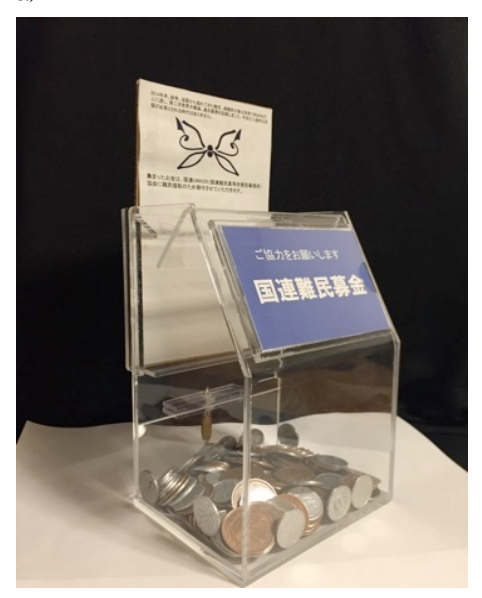

Figure 1.

a) The collection box of small-norm condition with control stimulus. b) The collection box of large-norm condition with eyes stimulus. 
a) Small-norm condition

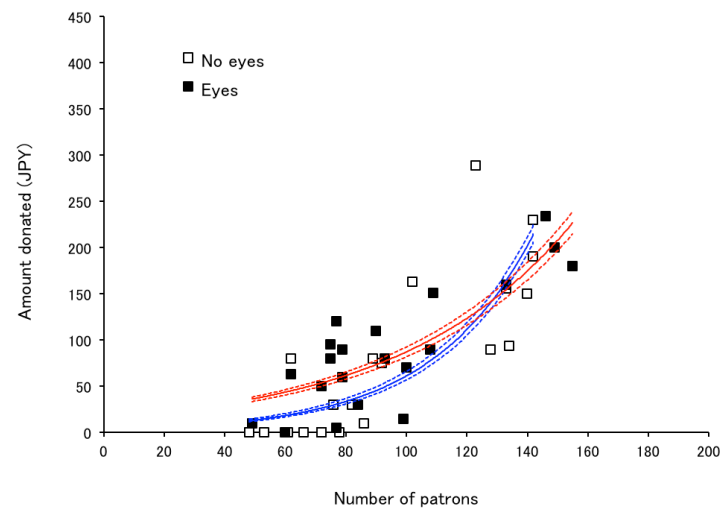

b) Large-norm condition

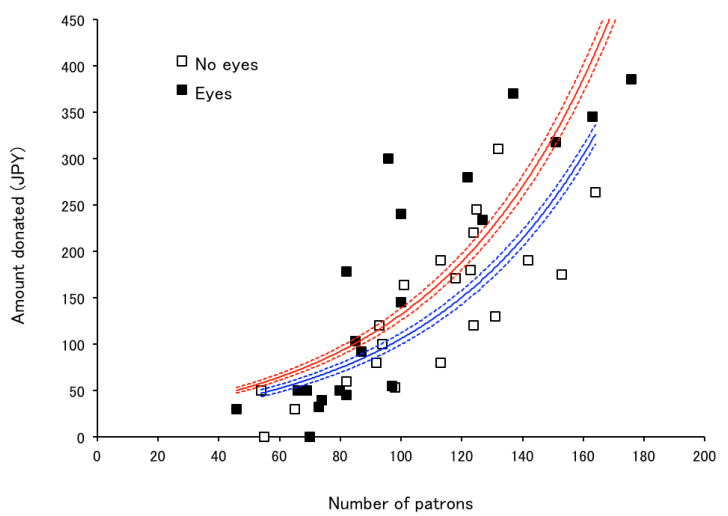

Figure 2.

Amount of donation by number of patrons in the izakaya, for eye and control images. Each point is an experimental day. Regression line is shown in solid blue (no eyes) and solid red (eyes) lines. The $95 \%$ confidence interval consists of the space between the two dotted lines. a) Small-norm condition. b) Large-norm condition.

amount donated each day as the response variable and the number of patrons who visited the izakaya each day, the presence of watching eyes, and their interaction as the predictors. We analyzed days under the small-norm $(n=42)$ and large-norm $(n=41)$ conditions separately because there was a significant interaction between norm and watching eyes such that the watching eyes effect was more salient under the small- than under the large-norm condition (Oda \& Ichihashi, 2016).

Under the small-norm condition $(A I C=1517.0)$, the main effects of the number of patrons and eyes were significant (patrons: $B=0.029, S E=0.001, z=31.665, p$ $<.001$; eyes: $B=1.588, S E=0.143, z=11.141, p<.001)$, as was the interaction between patrons and eyes $(B=$ $-0.012, S E=0.001, z=-10.550, p<.001)$. Regression lines were calculated for the amount of donation each day in each condition (Figure 2a). More money was donated when more patrons visited the izakaya. Furthermore, the presence of watching eyes increased the overall amount donated; however, the effect of the eyes image was more salient when fewer patrons visited the izakaya. When fewer patrons visited in the izakaya, regression line of the eyes condition was above that of the control condition, while the gap was reducing in response to the number of patrons. To clarify the interaction effect, we compared the amount donated under the eyes condition with that donated under the control condition using the median split of the number of patrons. When the number of patrons was below the median (range: $48-84 ; n=21$ ), the amount donated under the eyes condition $(n=11$, median: 60 JPY) was significantly greater than that donated under the control condition $(n=10$, median: 0 JPY; Wilcoxon rank-sum test: $W=18 ; p=.008)$. When the number of patrons was above the median (range: $86-155 ; n=21$ ), no significant difference was found between the amount donated under the eyes ( $n=11$, median: $130.5 \mathrm{JPY})$ and that donated under the control condition $(n=10$, median: $150 \mathrm{JPY} ; W=57 ; p=.915)$. Under the large-norm condition $(A I C=1616.6)$, the main effect of number of patrons was significant $(B=0.076, S E=0.004, z=21.416$, $p<.001)$; however, the main effect of watching eyes and the interaction were not (eyes: $B=-0.135, S E=0.156, z=$ $-0.869 ; p=.385$; interaction: $B=0.005, S E=0.004, z=$
1.087; $p=.277)$. Regression lines were calculated for the amount of donation each day in each condition (Figure 2b). More money was donated when more patrons visited the izakaya, but the presence of watching eyes did not affect the amount donated. Two regression lines did not intersect.

\section{Discussion}

We found that the presence of fewer people in the vicinity increased the effect of stylized eyes on charitable donations. Our findings are consistent with those of the two previous studies (Ekström, 2012; Powell et al., 2012) in which the subjects were customers in a shop. Our study extends the effect to patrons of a restaurant. A common factor among these studies is that they were conducted indoors where patrons could interact with each other (several may have visited as a group). If the watching eyes effect is situation specific, as Bateson et al. (2013) argued, then onthe-street charity collection using a box with an eyes stimulus might yield different results.

It may be that the eyes effect was less salient when the izakaya was crowded simply because the eyes stimulus was less conspicuous. When several patrons were in the izakaya, the collection box may have been difficult to see, or the patrons may have been distracted by the noisy atmosphere and not attended to the box. However, if the collection box was difficult to see in the crowded venue, it would follow that money donated would decrease as the number of patrons increased. To the contrary, we found that the amount donated was positively correlated with the number of patrons, suggesting that the patrons saw the collection box at a fixed frequency despite the congestion. Moreover, it is unlikely that the patrons did not notice the stylized eyes attached to the collection box. Our findings suggest that the effect of the watching eyes stimulus was similar to that of real eyes. The enhancing effect on prosociality may decline when patrons become habituated to being watched by "real" eyes.

\section{Acknowledgements}

This work was supported by JSPS KAKENHI Grant Number 25501003. 


\section{References}

Bateson, M., Callow, L., Holmes, J. R., Redmond Roche, M. L., \& Nettle, D. (2013). Do images of 'Watching Eyes' induce behaviour that is more pro-social or more normative? A field experiment on littering. PLoS ONE, 8: e82055. (doi: 10.1371/journal.pone.0082055)

Bateson, M. Robinson, R., Abayomi-Cole, T., Greenlees, J., O’Connor, A., \& Nettle, D. (2015). Watching eyes on potential litter can reduce littering: evidence from two field experiments. PeerJ, 3: e1443. (doi: 10.7717/ peerj.1443)

Ekström, M. (2012). Do watching eyes affect charitable giving? Evidence from a field experiment. Experimental Economics, 15, 530-546. (doi: 10.1007/s10683-0119312-6)

Ernest-Jones, M., Nettle, D., \& Bateson, M. (2011). Effects of eye images on everyday cooperative behavior: a field experiment. Evolution and Human Behavior, 32, 172-178. (doi: 10.1016/j.evolhumbehav.2010.10.006)

Fathi, M., Bateson, M., \& Nettle, D. (2014). Effects of watching eyes and norm cues on charitable giving in a surreptitious behavioral experiment. Evolutionary Psychology, 12, 878-887. (doi: $10.1177 / 147470491401200502)$

Matsugasaki, K., Tsukamoto, W., \& Ohtsubo, Y. (2015). Two failed replications of the watching eyes effect. Letters on Evolutionary Behavioral Science, 6, 17-20. (doi: 10.5178/lebs.2015.36)

Northover, S. B., Pedersen, W. C., Cohen, A. B., \& Andrews, P. W. (in press). Artificial surveillance cues do not increase generosity: two meta-analyses. Evolution and Human Behavior. (doi: 10.1016/ j.evolhumbehav.2016.07.001)

Oda, R., \& Ichihashi, R. (2016). Effects of eye images and norm cues on charitable donation: A field experiment in an izakaya. Evolutionary Psychology, 14, 1474704916668874. (doi: 10.1177/1474704916668874)

Oda, R., Kato, Y., \& Hiraishi, K. (2015). The watching-eye effect on prosocial lying. Evolutionary Psychology, 13 , 1474704915594959. (doi: 10.1177/1474704915594959)

Oda, R., Niwa, Y., Honma, A., \& Hiraishi, K. (2011). An eye-like painting enhances the expectation of a good reputation. Evolution and Human Behavior, 32, 166171. (doi: 10.1016/j.evolhumbehav.2010.11.002)

Powell, K. L., Roberts, G., \& Nettle, D. (2012). Eye images increase charitable donations: Evidence from an opportunistic field experiment in a supermarket. Ethology, 118, 1096-1101. (doi: 10.1111/eth.12011) 